\title{
LA INFINITUD ACTUAL DE PARTES DEL CONTINUO EN LA THEORIA MOTUS ABSTRACTI DE LEIBNIZ
}

\section{THE ACTUAL INFINITUDE OF PARTS OF THE CONTINUUM IN LEIBNIZ'S THEORIA MOTUS ABSTRACTI}

\author{
Federico Raffo Quintana ${ }^{1}$ \\ (IESCT-UNQ / CONICET)
}

Recibido: 30-7-2015

Aceptado: 8-2-2016

\begin{abstract}
Resumen: En la Theoria motus abstracti (TMA) de 1671 Leibniz afirmó, sin introducir mayores precisiones, que en el continuo hay infinitas partes en acto. Algunos exégetas entienden que las partes actuales han de entenderse como 'indivisibles'. En este trabajo sostendremos que puede defenderse otra interpretación que evita los problemas que tiene la de aquellos intérpretes y que se esclarece sobre la base de los exámenes aritméticos de Leibniz inmediatamente posteriores a la redacción de la TMA. Así, mostraremos que habría un paralelismo entre los exámenes de Leibniz sobre el problema continuo y sobre series infinitas.
\end{abstract}

Palabras clave: Infinito actual; continuo; indivisibles; series infinitas.

\begin{abstract}
In his 1671's Theoria motus abstracti (TMA), Leibniz stated without further precisions that there is an actual infinity of parts in the continuum. Some exegetes hold that the actual parts must be understood as 'indivisibles'. In this paper we will hold that another interpretation can be defended, which avoids the problems that the other interpretation has, and which is clarified on the basis of Leibniz's arithmetical exams written immediately after the writing of the TMA. Thus, we will show that there could be a parallelism between Leibniz' exams on the continuum problem and on infinite series.
\end{abstract}

Key words: Actual infinite; continuum; indivisibles; infinite series.

[1] (federq@gmail.com)_Federico Raffo Quintana es Licenciado en Filosofía por la Universidad Católica Argentina (UCA). En su tesis de licenciatura abordó algunas problemáticas relativas al espacio, al tiempo y al movimiento en el marco del examen leibniziano del continuo. Actualmente se encuentra realizando el doctorado en la Universidad Nacional de la Plata (UNLP) centrándose en los conceptos de continuo e infinito en el pensamiento temprano de Leibniz, para lo cual cuenta con una beca del CONICET. 
Uno de los problemas a los que Leibniz dedicó mayor atención durante sus años de juventud es el problema de la composición del continuo. ${ }^{2}$ Este problema (que consiste, sucintamente, en el examen de una conjunción de cuestiones relativas a las magnitudes finitas, tales como si el número de partes en ellas es finito o infinito, si dichas partes, en el caso de que sean infinitas en número, se dan actual o potencialmente, si son indivisibles o no, etc.) generó un gran interés en pensadores de la temprana filosofía moderna tales como G. Galileo (1564-1642), ${ }^{3}$ R. Descartes (1595-1650), ${ }^{4}$ P. Gassendi (1592-1655), ${ }^{5}$ y L. Froidmont (1587-1653), ${ }^{6}$ entre muchos otros. Una de las primeras manifestaciones del interés de Leibniz por el problema del continuo se encuentra en un escrito que presentó a la Ilustre Academia Real de Francia en el año 1671, esto es, la Theoria motus abstracti (Teoría del movimiento abstracto, de aquí en más TMA). Este fue el primer trabajo en el que Leibniz abordó ordenadamente el problema del movimiento, por lo que es un antecedente de lo que, tras muchos años de análisis, será su dinámica. Su preocupación por la teoría del movimiento

[2] Para una presentación del abordaje leibniziano del problema del continuo recomendamos los múltiples trabajos de Richard Arthur, como The Labyrinth of the Continuum. Writings on the Continuum Problem, 1672-1686, textos seleccionados, traducidos y editados e introducción elaborada por Richard T. W. Arthur, New Haven y Londres: Yale University Press, 2001, introducción; "Leibniz on Continuity", en PSA: Proceedings of the Biennial Meeting of the Philosophy of Science Association, 1, 1986, pp. 107-115; "Cohesion, Division and Harmony: Physical Aspects of Leibniz's Continuum Problem (1671-1686)”, en Perspectives of Science, 6 nos. 1 \& 2, 1998, pp. 110-135; "Leibniz's Inversion of Zeno: Continuity of Motion, Substantial Action and Plurality", en Workshop 'Corporeal Substances and the Labyrinth of the Continuum' (2000), http://www.humanities.mcmaster.ca/ rarthur/papers/LIZ.pdf [Consultado: 10/02/2016]; Actual Infinitesimals in Leibniz's Early Thought", en The Philosophy of the Young Leibniz, Studia Leibnitiana, Sonderheft 35, 2009, pp. 1-26. Asimismo, pueden verse los siguientes trabajos: Bassler, Otto Bradley, "Leibniz on the Indefinite as Infinite", en The Review of Metaphysics, 51, 4, 1998, pp. 849-874; Bassler, Otto Bradley, "The Leibnizian Continuum in 1671", en Studia Leibnitiana, 30, 1, 1998, pp. 1-23; Lawrenz, Jürgen, The Nature of Reality and the Reality of Nature. A Study of Leibniz's Double-Aspect Ontology and the Labyrinth of the Continuum, Cambridge: Cambridge Scholars Publishing, 2010. Finalmente, para un examen general más completo del tratamiento leibniziano del problema del continuo de la Theoria motus abstracti puede verse Beeley, Philip, Kontinuität und Mechanismus. Zur Philosphie des jungen Leibniz in ihrem Ideengeschichtlichen Kontext, Studia Leibnitiana Supplementa, vol. 30, Stuttgart: Franz Steiner Verlag, 1996.

[3] Galileo aborda el problema del continuo especialmente en sus Discorsi. EN VIII, 49-150.

[4] Pueden hallarse algunas aproximaciones de Descartes a este problema en la primera y especialmente la segunda parte de los Principia philosophiae. AT, VIII, 5-79.

[5] Véase Gassendi, Pierre, Opera omnia in sex tomos divisa, Lvgdvni: Sumptibus Lavrentii Anisson \& Ioan. Bapt., Devenet, 1658, Vol. I, Par. 2, Lib. 3, 229-282.

[6] Este autor ha dedicado una obra al problema del continuo. Froidmont, Libert, Labyrinthus sive de compositione continui, Anvers, 1631.

Thémata. Revista de Filosofía $\mathrm{N}^{\circ} 53$ (2016) pp.: 289-310. 
surge a raíz de un trabajo del científico holandés C. Huygens (1629-1695) publicado en Philosophical Transactions (trabajo que Leibniz conoció gracias a Erich Mauritius) sobre las reglas de choque que escribió animado por la Real Academia de Londres. Por eso mismo, la TMA inicialmente formaba parte de un trabajo único junto con la Hypothesis physica nova de 1671 que finalmente Leibniz envió a la Real Academia de Londres. Como Leibniz había establecido relaciones con uno de los miembros de la Academia de París, Pierre de Carcavy (1600-1684), decidió separar los escritos y enviar uno a cada una de las dos academias (A VI 2, XXXI-XXXIII y OFC 8, 73). Aunque los exámenes de Leibniz de la TMA hayan ido profundizándose y muchas veces evolucionando en los años posteriores, se trata de una obra de gran importancia en el pensamiento de juventud del autor. Esto se debe, por una parte, a que es su primer intento de abordar las razones universales de los movimientos al margen de las sensaciones y los fenómenos. ${ }^{7}$ Asimismo, por otra parte, allí Leibniz presentó por primera vez un examen más o menos sistemático sobre el problema de la composición del continuo. En una carta que Leibniz le envió a Pierre de Carcavy muy poco después de haber finalizado la redacción de la $T M A$ se encuentra un resumen de los principales temas considerados, así como la ubicación de los respectivos tratamientos dentro de este escrito:

En la Teoría del movimiento abstracto se ha desarrollado: el laberinto de la composición del continuo, fund. predemost. 1-5, fund. 18; una demostración de la verdad de la Geometría de los indivisibles de Cavalieri, fund. 5, que hasta ahora solo había sido admitida con el valor de una hipótesis, porque de ella, si se la utiliza correctamente, pueden deducirse muchas verdades y ningún absurdo; la verdadera naturaleza del conato, fund. 6-16; la primera e íntima razón de la cohesión o firmeza elaborada con certeza geométrica, fund. 15, 16, teor. 17; la verdadera distinción hasta ahora no considerada de la mente respecto del cuerpo, fund. 17; un examen de la discutida igualdad entre el ángulo de incidencia y el de reflexión, $t h$. 7; una construcción física, esto es, exacta y al mismo tiempo real de las cosas geométricas, en los problem.; producir una curva a partir de meros movimientos rectos y viceversa, probl. 6 , 7; producir un cilindro, un cono, una esfera a partir de meros cuerpos rectilíneos, probl. 17, 18, 19; producir cuerpos de secciones cónicas, probl. 20; acelerar y retardar continuamente un movimiento dado, probl. 24, 25». (A II 1, 236-237. La traducción es nuestra)

Como se ve, este repaso trasluce la centralidad del problema del continuo en la TMA. En la sección en la que se encuentra el abordaje del problema del continuo, los fundamenta praedemonstrabilia, Leibniz comienza afirmando sin rodeos que «(1) [s]e dan en acto partes en el continuo, contra lo que piensa el agudísimo Thomas White, (2) y ellas son infinitas en acto;

[7] Precisamente el título completo del escrito es «Teoría del movimiento abstracto, es decir, razones universales de los movimientos independientes de los sentidos y de los fenómenos» (A VI 2, 259).

Thémata. Revista de Filosofía $\mathrm{N}^{\circ} 53$ (2016) pp.: 289-310. 
en efecto, lo indefinido de Descartes no existe en la cosa sino en el pensante» (A VI 2, 264). ${ }^{8} \mathrm{Si}$ bien no lo explica en este escrito, se desprende de algunos textos anteriores que, de acuerdo con Leibniz, la tesis cartesiana según la cual la división de la materia es «al infinito, o indefinida», esto es, «en tantas partes que no podamos pensar en ninguna que no sea tan diminuta, que entendamos que no está dividida realmente en otras todavía más pequeñas» (AT, VIII, $2, \S 33),{ }^{9}$ implica el absurdo de atribuir a la materia una propiedad (esto es, la indefinición) que en rigor depende del pensamiento (a saber, del hecho de no entender que existen partes más pequeñas). En este sentido, para Leibniz la indefinición no es algo que esté en la realidad, donde se encuentran partes actuales e infinitas, sino a lo sumo en la mente de quien piensa (A VI 2, 279-280; A II 1, 14-38, entre otros).

Ahora bien, una de las grandes dificultades que se encuentra en la $T M A$ es que Leibniz no ha explicado cómo han de entenderse las infinitas partes actuales. En este sentido, se ha limitado simplemente a enunciar los fundamentos predemostrables. En general, los especialistas consideran que Leibniz habría entendido que las infinitas partes actuales son «indivisibles». Así lo entienden, por ejemplo, Bassler, ${ }^{10} \operatorname{Lison}^{11}$ y Arthur en algunas ocasiones, ${ }^{12}$ aunque en otras ha cuestionado esta interpretación. ${ }^{13} \mathrm{Esta}$ interpretación se sustenta en el hecho de que en el cuarto fundamento predemostrable de la TMA Leibniz postula indivisibles:

Se dan los indivisibles o inextensos, de otro modo no puede entenderse ni el inicio ni el fin del movimiento o del cuerpo. La demostración es esta: se da el inicio y el fin del algún espacio, cuerpo, movimiento, tiempo; sea aquello cuyo inicio se busca expuesto en la línea $a b$, cuyo punto medio es $c$, y sea $d$ el medio entre $a$ y $c$ y $e$ entre $a$ y $d$, y así sucesivamente. Búsquese el inicio por la izquierda, en el lado $a$. Digo que $a c$ no es el inicio, puesto que puede quitársele $d c$ conservado el inicio; ni $a d$ [es el inicio] puesto que

[8] Tomamos las traducciones de esta obra de OFC 8: 73-95, aunque en ocasiones introducimos modificaciones. En el prefacio que hizo para la obra Demonstratio inmortalitate animae rationalis (1664) de Kenelm Digby (1603-1665), que Leibniz conocía al menos desde 1668 (A VI 1, 501 y ss.), Thomas White señaló que el continuo es una cierta entidad unitaria, es decir, un todo cuyas partes son sólo potenciales, pero nunca en acto.

[9] Traducciones López, E. y Graña, M., Sobre los principios de la filosofía (traducción y notas), Madrid: Gredos, 1989.

[10] Bassler, Otto Bradley, “The Leibnizian Continuum in 1671”, op. cit.

[11] Lison, Elad, "The Philosophical Assumptions Underlying Leibniz's Use of the Diagonal Paradox in 1672", en Studia Leibnitiana, 38, 2, 2006, pp. 197-208.

[12] Arthur, Richard T. W., "Leibniz on Continuity", op. cit., 108-109; "Cohesion, Division and Harmony", op. cit., 113; The Labyrinth of the Continuum, op. cit., xxxii-xxxvii; "Actual Infinitesimals in Leibniz's Early Thought", op. cit., 9-17.

[13] Arthur, Richard T. W., "Leibniz's Inversion of Zeno: Continuity of Motion, Substantial Action and Plurality", op. cit., 7.

Thémata. Revista de Filosofía N53 (2016) pp.: 289-310. 
puede quitarse ed [conservado el inicio], y así sucesivamente. Por consiguiente, no es un inicio algo a lo cual puede quitarse [una parte] a la derecha. [Algo] a lo cual no puede quitarse extensión es inextenso. Por consiguiente, el inicio del cuerpo, del espacio, del movimiento, del tiempo (el punto, el conato, el instante) o no es nada, lo que es absurdo, o es inextenso, que era lo que debía demostrarse». (A VI 2, 264)

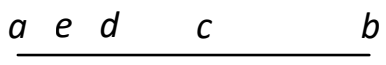

Leibniz se ha valido del concepto de lo indivisible, que como se observa fue presentado en la TMA apenas después de haber afirmado que se dan infinitas partes actuales en el continuo, especialmente para explicar, en términos abstractos, algunos problemas físicos relativos al movimiento y al cuerpo. Sintéticamente, en lo que respecta al movimiento, Leibniz explicó que la desigualdad de las velocidades de los movimientos se funda en la diferencia de las magnitudes de los respectivos conatos indivisibles, así como también, en lo que respecta al cuerpo, explicó que ellos están cohesionados puesto que sus partes tienen puntos indivisibles extremos en común (A VI 2, 265-267). ${ }^{14}$ Más aún, la palabra «indivisible» tuvo una importante presencia en numerosos trabajos tanto matemáticos como filosóficos de la época (aunque no siempre se ha entendido lo mismo por dicho término). Por ejemplo, en el célebre trabajo del matemático italiano Bonaventura Cavalieri (1598-1647) Geometria indivisibilibus continuorum quadam nova ratione promota de 1635, del que Leibniz tenía algún conocimiento en la época de la $T M A$ presumiblemente por medio de la crítica de Hobbes, ${ }^{15}$ o bien la teoría de una composición del continuo de indivisibles non quanti sostenida por Galileo en los Discorsi e dimostrazione matematiche, intorno a due nuove scienze de 1638 que Leibniz leyó luego de presentar la TMA en 1672 (A VI 3, 163-168). El hecho de que Leibniz haya leído la obra de Galileo en 1672, por cierto, significa que el filósofo de Leipzig no se limitó a enunciar las ideas del científico italiano, sino que incluso ha cuestionado $\mathrm{su}$ indivisibilismo, por ejemplo, en De minimo et maximo. De corporibus et mentibus (A VI 3, 97-98). De acuerdo con Bassler, en la TMA Leibniz habría tomado la idea de una "composición de indivisibles» del método de

[14] Con respecto al examen del movimiento, véase nuestro trabajo «Indivisibles y movimiento en el continuo leibniziano» que se publicará próximamente en el número 45 del año 2015 de la Revista Latinoamericana de Filosofía.

[15] Beeley, Philip, "Infinity, Infinitesimals and the Form of Cavalieri: John Wallis and his Critics", en Ursula Goldenbaum y Douglas Jesseph eds., Infinitesimal Differences: Controversies between Leibniz and his Contemporaries, Berlin y New York: Walter de Gruyter, 2008, pp. 44-45.

Thémata. Revista de Filosofía N53 (2016) pp.: 289-310. 
Cavalieri. ${ }^{16}$ Esta opinión descansa en el hecho de que Leibniz se ha referido a dicho método en la TMA. En efecto, en el quinto fundamento predemostrable el filósofo de Leipzig presentó una noción de punto, como de aquello cuya magnitud es inconsiderable o inasignable, que a su decir es «(...) el fundamento del Método Cavaleriano (...)» (A VI 2, 265). ${ }^{17}$ Leibniz reiteradamente indica que el problema del continuo, cuya dilucidación es uno de los objetivos de la TMA, es también importante (entre otras cosas) en vistas a ubicar en bases sólidas tanto la geometría de los indivisibles (haciendo referencia al pensamiento de Cavalieri) como la aritmética de los infinitos (refiriéndose, presumiblemente, al de John Wallis) (A VI 2, 262). Esto se ha observado también en el resumen de los principales lineamientos de la TMA que Leibniz le ha enviado en una carta a Carcavy y que mencionamos anteriormente.

No obstante, sostener que en la TMA Leibniz ha entendido que las infinitas partes actuales del continuo son indivisibles es problemático. La razón principal para decir esto es que Leibniz nunca identificó explícitamente las infinitas partes con los indivisibles ni tampoco mencionó que el continuo se compone de ellos. Este importante hecho ha sido señalado incluso por algunos estudiosos del pensamiento de Leibniz que sostienen la interpretación antes mencionada, como Arthur y Bassler. ${ }^{18}$ Más aún, es posible que Leibniz nunca haya querido decir que las infinitas partes actuales de la TMA deben tomarse como los indivisibles que postula en la misma obra. Un indicio de esto se encuentra en un escrito preparatorio para la TMA en que Leibniz ha dicho que una división hasta los indivisibles es imposible. El contexto en el que ha afirmado la imposibilidad de una división hasta los indivisibles es, a su vez, muy importante. Desde 1669, Leibniz estuvo trabajando los temas que dieron lugar a la versión definitiva de la TMA.

[16] Bassler, Otto Bradley, "The Leibnizian Continuum in 1671”, op. cit., p. 8.

[17] Leibniz se aleja, así, de la manera como han entendido la noción de punto Euclides en el libro I de Elementa (esto es, «[u]n punto es lo que no tiene partes», traducción de María Luisa Puertas Castaños) y Hobbes en De corpore, II, 8, 12. Sobre esta noción leibniziana de punto, véanse, entre otros, Arthur, R. "Actual Infinitesimals in Leibniz's Early Thought", op. cit. pp. 9-17 y Bernstein, Howard, "Conatus, Hobbes, and the young Leibniz", en Studies in History and Philosophy of Science, 11, 1, 1980, pp. 25-37. Sobre el método de Cavalieri, véanse Andersen, Kirsti, "Cavalieri's Method of Indivisibles", en Archive for History of Exact Sciences, 31, 1985, pp. 291-367 y Andersen, Kirsti, "The Method of the Indivisibles: Changing Understandings", en Studia Leibnitiana, Sonderheft 14, 1986, pp. 14-25.

[18] Arthur, R., "Actual Infinitesimals in Leibniz's Early Thought”, op. cit., p. 10, y Bassler, O. B., "The Leibnizian Continuum in 1671", op. cit., p. 8. Distinto es el caso de Lison, E., "The Philosophical Assumptions Underlying Leibniz's Use of the Diagonal Paradox in 1672", op. cit., para quien Leibniz habría explícitamente afirmado que el continuo se compone de puntos indivisibles, de modo que las infinitas partes actuales deberían tomarse como indivisibles, en un pasaje de una carta que le envió a Oldenburg en abril de 1671 (A II 1, 166). No obstante, el pasaje al que refiere Lison no confirma su aserción.

Thémata. Revista de Filosofía No53 (2016) pp.: 289-310. 
En uno de estos trabajos, Leibniz se pregunta si dos cuerpos que chocan entre sí se unen o no y, en el caso de que se unan, si lo hacen en un punto, una línea o una cara (hedra). Leibniz reconoce que, si se supone que los cuerpos que chocan se unen en un punto o una línea, entonces ellos inevitablemente se dividirán hasta los puntos o líneas. Es en este contexto que Leibniz menciona que:

(...) si las cosas que concurren en un punto se unen, es necesario que la división suceda ininterrumpidamente hasta los puntos. Y si concurren en una línea, es necesario que la división de las partes suceda ininterrumpidamente hasta las líneas; pero tal división ininterrumpidamente hasta los indivisibles es, en realidad, imposible».

(A VI 2, 165)

De esta manera, este pasaje mostraría que la interpretación recién mencionada no es acertada. A esto hay que sumarle que, en los pasajes en los que se refiere al método de Cavalieri, Leibniz no dice que ha tomado la idea de una composición de indivisibles de aquel método sino que cree haber ofrecido un fundamento para él. En este sentido, la relación entre el método de Cavalieri y la teoría leibniziana del continuo es exactamente inversa a la sostenida por Bassler. Esta situación lleva a un gran inconveniente. En efecto, ¿cómo entender las infinitas partes actuales, si no han de concebirse como indivisibles? ¿Es posible pensar la infinitud actual de partes de TMA de otra manera? Aunque a nuestro modo de ver no sea acertado pensar que en la TMA Leibniz tomó la idea de una composición de indivisibles del método de Cavalieri, en este planteo de Bassler se halla subyacentemente presente la visión de que el examen leibniziano del continuo de la TMA está conectado con la matemática. Algunos especialistas en el pensamiento de Leibniz señalan incluso que el examen del continuo, especialmente el físico, tiene un 'núcleo matemático'. ${ }^{19}$ En lo siguiente buscaremos argumentar que la manera como Leibniz entendió una infinitud actual de partes en la TMA podría esclarecerse a partir de su trabajo matemático posterior, especialmente sobre aritmética de los infinitos. Dicho de otro modo, buscaremos justificar que la tesis de la infinitud actual de partes de la $T M A$ podría aclarase a partir del trabajo de Leibniz sobre series infinitas. Así, Leibniz habría comprendido que el infinito actual obedece a un orden determinado, por lo que las partes, que en las series infinitas están representadas en las fracciones, no pueden ser escogidas o asignadas arbitrariamente.

Como es sabido, Leibniz no tuvo grandes conocimientos técnicos de matemática hasta su llegada a París en 1672, a pesar de haberse interesado por algunas cuestiones más bien epistemológicas, como por el método

[19] Beeley, Philip, "Mathematics and nature in Leibniz's early philosophy", en Brown, Stuart ed., The young Leibniz and his philosophy (1646-76), Dordrech/Boston/London : Kluwer Academic Publishers, 1999, pp. 123-145.

Thémata. Revista de Filosofía $\mathrm{N}^{\circ} 53$ (2016) pp.: 289-310. 
deductivo de la matemática y por el aspecto formal de la computación (por ejemplo, en su Dissertatio de arte combinatoria de 1666). En sus años escolares había tenido apenas contacto con el primer libro de los Elementos de Euclides (incluso, posiblemente con una versión abreviada) y con algunos trabajos aritméticos como el de Ch. Clavius (1537-1612) publicado en 1585, Epitome arithmetica practica, o el de Jo. Lanz (1564-1638) editado en 1616, Institutiones arithmeticae. Una vez graduado amplió un poco sus conocimientos, pero sin lograr gran profundidad. ${ }^{20}$ Poco después de haber llegado a la capital francesa en 1672 Leibniz conoció a Christiaan Huygens, bajo cuya tutela comenzó a trabajar en los aspectos más bien técnicos de la matemática, especialmente de la aritmética. En efecto, uno de los primeros desafíos que el científico holandés le propuso a Leibniz fue que llevara a cabo la suma de la serie de los recíprocos de los números triangulares (esto es, $1 / 1,1 / 3,1 / 6,1 / 10$, etc.) o sea, de una serie infinita (A II 1, 344). No obstante, los resultados del trabajo de Leibniz fueron mucho más allá, pues además de cumplir con las expectativas de Huygens propuso una regla universal para la suma de series infinitas (A II 1, 345). A raíz de esto, Leibniz redactó un trabajo en el que sintetizó los resultados de sus primeras investigaciones matemáticas y se lo envió al entonces director del Journal des Sçavans, Jean Gallois, para ser publicado (aunque finalmente no lo haya sido por motivos ajenos a Leibniz). En este escrito, titulado Accessio ad arithmeticam infinitorum, es decir, Introducción a la aritmética de los infinitos (de aquí en más Accessio), Leibniz llevó a cabo un examen sobre series infinitas que nos permitirá representarnos la manera como habría entendido anteriormente, en la TMA, una infinitud actual de partes. En efecto, como veremos en estas series hay [a] infinitos términos (es decir, no hay un último asignable) [b] generados según un orden (esto es, según la ley de la serie) [c] que, sumados -excepto en algunos casos que mencionaremos más adelante-, dan lugar a un todo finito. En consonancia, las infinitas partes actuales del continuo de TMA podrían entenderse como los términos de la serie que no pueden ser escogidos arbitrariamente y que, agregados, dan lugar a una cierta totalidad finita.

[20] Hofmann, Joseph E., Leibniz in Paris, 1672-1676. His growth to mathematical maturity, Cambridge y New York: Cambridge University Press, 1974, pp. 1-11.

Thémata. Revista de Filosofía ${ }^{\circ} 53$ (2016) pp.: 289-310. 
Decir que Leibniz analiza el infinito en sus exámenes aritméticos no significa ni que haya sido especialmente novedoso en este terreno más allá de la regla para la suma de series a la que hemos aludido anteriormente ni mucho menos que haya sido el primero en considerar estos asuntos. Más aún, Leibniz reconoce que la aritmética de los infinitos fue revitalizada en su tiempo por el matemático británico John Wallis (1616-1703). La historia del tratamiento matemático del infinito remite incluso hasta el pensamiento de la antigua Grecia. En algún sentido, el punto de partida de esta historia puede hallarse en el método de exhaución de Arquímedes (que fue el resultado de un perfeccionamiento del de Eudoxo). En los siglos XVI y XVII han sido fundamentales los trabajos de L. Valerio (1553-1618), J. Kepler (1571-1630), B. Cavalieri, G. Galilei, Grégoire de Saint-Vincent (15841667), Gilles Personne de Roberval (1602-1675), entre muchos otros. ${ }^{21} \mathrm{~A}$ su vez, el infinito ha sido objeto de un tratamiento no matemático sino filosófico fundamentalmente desde Aristóteles y el pensamiento escolástico del siglo XIII en adelante, destacándose por ejemplo la discusión sobre el problema del continuo, el infinito y los indivisibles y sobre la actualidad o potencialidad del infinito en autores como T. Bradwardine (1290-1349), G. de Ockham (ca. 1280-ca. 1349), o posteriormente N. de Cusa (1401-1464). ${ }^{22}$ En el siglo de Leibniz, además de la revitalización de la aritmética de los infinitos por parte de Wallis, merecen una mención especial los exámenes de Pascal en relación con su Traité du triangle arithmétique de 1665. Como hemos dicho, lo que aquí nos interesa es mostrar que el examen leibniziano sobre series infinitas de 1672 permite que nos representemos la manera como Leibniz habría entendido la infinitud actual de partes del continuo en la TMA. Ahora bien, en la Accessio (escrito al que nos hemos referido anteriormente como aquel del que nos valdremos para examinar el tratamiento de Leibniz sobre series infinitas) el autor tiene en cuenta dos tipos de series, según los procedimientos que determinan su generación. Por este motivo, a continuación consideraremos por separado estas dos variantes, refiriéndonos a ellas como 'dos modelos', para mostrar posteriormente que los escritos sobre el problema del continuo, tanto del año

[21] Una aproximación al planteo y a la relevancia histórica de estos autores puede encontrarse en Boyer, Carl B., The history of the calculus and its conceptual developement, New York: Dover Publications, Inc., 1949, capítulos 2 y 4.

[22] Similarmente a lo que hemos indicado en la nota anterior, véase Boyer, C., The history of the calculus and its conceptual developement, op. cit., capítulo 3.

Thémata. Revista de Filosofía No53 (2016) pp.: 289-310. 
en que redactó la Accessio como del año anterior, se valen especialmente de uno de ellos.

El primer modelo mediante el cual podemos representarnos que Leibniz habría entendido una infinitud de partes actuales es el de series «decrecientes con una progresión Geométrica al infinito» (A II 1, 343). ${ }^{23}$ Una serie de este tipo es aquella en la que las partes son proporcionales, esto es, están divididas según una ratio que permanece constante. ${ }^{24} \mathrm{En}$ este sentido, en estas series una subsección es a una sección lo que ella es al todo (A II 1, 343). ${ }^{25} \mathrm{~A}$ modo de ejemplo, Leibniz expone en una línea una serie de fracciones tales que cada una sea una tercera parte de la anterior (de manera que la proporción sea la de un tercio):

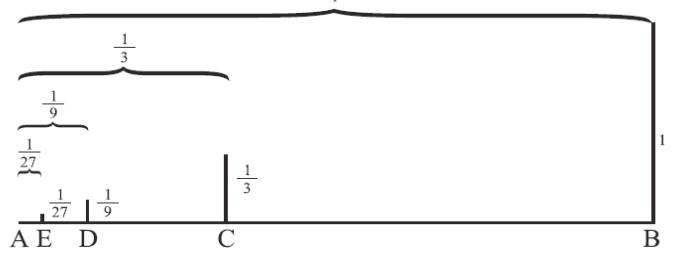

El hecho de que Leibniz exponga estas series en líneas será muy significativo para el problema del continuo. Por una cuestión de orden, examinaremos esto un poco más adelante, luego de presentar los dos modelos de series. En este ejemplo de Leibniz se observa la propiedad mencionada anteriormente, es decir, que entre una sección y el todo se mantiene la misma ratio que entre una subsección y una sección, de tal modo que el todo $\mathrm{AB}$ es a $\mathrm{AC}$ proporcionalmente lo mismo que $\mathrm{AC}$ a $\mathrm{AD}$, y así sucesivamente, esto es: $A B / A C=A C / A D=A D / A E=$ etc. En este ejemplo particular, cada subsección representa una tercera parte de la sección en la cual se incluye. De esta manera, $\mathrm{AC}$ es $1 / 3 A B$, así como $\mathrm{AD}$ es $1 / 3 A C$ y por eso mismo $1 / 9 A B$, del mismo modo que $\mathrm{AE}$ es $1 / 3 A D$ y $1 / 27 A B$, y así sucesivamente.

Ahora bien, en la Accessio Leibniz abordó también otro tipo de series que en cuanto tales expresan otro modelo con el cual habría entendido una infinitud actual de partes. En este caso, no se trata de series que decrecen según una progresión geométrica al infinito sino de series que decrecen, utilizando una expresión de Leibniz, según los recíprocos de «alguna progresión aritmética replicada» (A II 1,345-346). Un ejemplo de

[23] Todas las traducciones de esta obra son nuestras.

[24] Para denominarlas de esta manera tenemos en cuenta la noción de 'proporción' que Leibniz ha presentado algunos años más tarde: «Proportio est rationum aequalitas». A VI 3, 483.

[25] Allí Leibniz explica esta propiedad y menciona el ejemplo que daremos a continuación.

Thémata. Revista de Filosofía №53 (2016) pp.: 289-310. 
series de este tipo es el que Huygens le planteó a Leibniz para que busque su suma, a saber, la serie de los recíprocos de los números triangulares. A decir de Leibniz, las «progresiones aritméticas replicadas» son las mismas series que Pascal ha expuesto en su Traité du triangle arithmétique de 1665, es decir, son las series del triángulo de Pascal: ${ }^{26}$

$\begin{array}{lrrrrrrrrrr}{[\mathrm{A}]} & 1 & 1 & 1 & 1 & 1 & 1 & 1 & 1 & 1 & 1 \\ {[\mathrm{~B}]} & 1 & 2 & 3 & 4 & 5 & 6 & 7 & 8 & 9 & \\ {[\mathrm{C}]} & 1 & 3 & 6 & 10 & 15 & 21 & 28 & 36 & & \\ {[\mathrm{D}]} & 1 & 4 & 10 & 20 & 35 & 56 & 84 & & & \\ {[\mathrm{E}]} & 1 & 5 & 15 & 35 & 70 & 126 & & & \\ {[\mathrm{~F}]} & 1 & 6 & 21 & 56 & 126 & & & & \\ {[\mathrm{G}]} & 1 & 7 & 28 & 84 & & & & & \\ {[\ldots]} & 1 & 8 & 36 & & & & & & & \\ & 1 & 9 & & & & & & & & \\ & 1 & & & & & & & & \end{array}$

Como puede observarse en la tabla, el triángulo expone números de distintos órdenes que se determinan según un procedimiento estipulado. En cualquiera de los órdenes, de acuerdo con Pascal, los términos de una serie son a su vez las diferencias de los términos de la serie del orden inmediatamente anterior. ${ }^{27}$ De esta manera, las unidades, que se observan en el primer nivel de la tabla, constituyen el primer orden (serie [A]), al mismo tiempo que son las diferencias de los términos del segundo orden. Dicho de otra manera, los números de segundo orden (serie [B]) serán aquellos que se forman por la adición de los números del primer orden, esto es, de unidades. De esta manera, los números de segundo orden serán los naturales, esto es, 1, 2, 3, 4, etc. Este mismo procedimiento puede replicarse al infinito, dando lugar a series de distinto orden. Así, luego de la de los números naturales se halla la serie de los triangulares (serie [C]), esto es, de los números que se forman por sumar los naturales (1, 3, 6, 10, 15, etc.), en siguiente lugar se encuentra la serie de los piramidales (serie [D]), los cuales números se forman por la adición de los triangulares $(1,4,10,20$,

[26] Pascal, Blaise, Traité du triangle arithmétique, avec quelques autres petits traitez sur la mesme matière, París: G. Desprez, 1665, pp. 2-3. Leibniz se refiere en A II 1, 345-346.

[27] Pascal, B., Traité du triangle arithmétique, op. cit. 2.

Thémata. Revista de Filosofía No53 (2016) pp.: 289-310. 
etc.), y así sucesivamente al infinito. Pascal sintetiza el resultado de este procedimiento en la siguiente tabla: ${ }^{28}$

\begin{tabular}{cc|cccccc} 
& \multicolumn{1}{c}{1} & 2 & 3 & 4 & 5 & Etc. \\
\cline { 3 - 5 } Unidades & Orden 1 & 1 & 1 & 1 & 1 & 1 & Etc. \\
Naturales & Orden 2 & 1 & 2 & 3 & 4 & 5 & Etc. \\
Triangulares & Orden 3 & 1 & 3 & 4 & 10 & 15 & Etc. \\
Piramidales & Orden 4 & 1 & 4 & 10 & 20 & 35 & Etc. \\
& Etc. & & & & & &
\end{tabular}

Ahora bien, como hemos dicho, las series para las cuales Leibniz propuso una regla de suma en la Accessio no son formalmente las expuestas por Pascal en su triángulo aritmético sino las de los recíprocos de dichas series. Utilizando una expresión que en rigor empleó unos años más adelante, a partir del 'triángulo aritmético' de Pascal Leibniz construye el de los recíprocos correspondientes y que llamó ‘triángulo armónico', esto es: ${ }^{29}$

Recíprocos de

Triángulo Armónico

Naturales

Triangulares

$1 / 1$

$1 / 2$

$1 / 3$

$1 / 4$

$1 / 5 \quad 1 / 6$

Piramidales

$1 / 1$

$1 / 3$

$1 / 6$

$1 / 10$

1/15 Etc

Triángulo-Triangulares １/1

$1 / 4$

$1 / 10$

$1 / 20 \quad$ Etc.

Triángulo-Piramidales

$1 / 5$

$1 / 15$

Etc.

Piramido-Piramidales

$1 / 1$

Etc.

Leibniz se refiere a este tipo de series como 'replicadas' porque, si se sustituyera la unidad como generador de la serie por otro número, se replicaría proporcionalmente toda la serie. Por ejemplo, si en vez de 1 el generador fuese 2 , la serie que se obtendría en el caso de las fracciones triangulares sería $2 / 2,2 / 6,2 / 12$ etc. Ahora bien, precisamente por decrecer según los recíprocos de alguna progresión aritmética replicada, estas series no decrecen con una progresión geométrica, es decir, sus términos no son

[28] Pascal, B., Traité du triangle arithmétique, op. cit. 3.

[29] Leibniz, G. W., Quadrature arithmétique du cercle, de l'ellipse et de l'hyperbole et la trigonométrie sans tables trigonométriques qui en est le corollaire (introducción, traducción y notas de Marc Parmentier, texto latino editado por Ebenhard Knobloch), París: Vrin, 2004, p. 230. Allí puede hallarse la tabla sobre la que basamos la que presentamos a continuación.

Thémata. Revista de Filosofía $\mathrm{N}^{\circ} 53$ (2016) pp.: 289-310. 
proporcionales. En esto difieren precisamente los dos modelos con los cuales podemos representarnos que Leibniz ha entendido una infinitud actual de partes. Sin embargo, entre estos dos modelos hay, como se ha dicho, una estructura común, pues en ambos la partición del todo debe responder a una cierta ley de sucesión tal que cada término sea menor que el anterior. Por eso mismo, en ambos modelos las fracciones no pueden ser escogidas o asignadas arbitrariamente.

Como hemos dicho al comienzo, los exámenes aritméticos de Leibniz son aquí importantes para observar el infinito presente en las series infinitas y en consecuencia para comprender, a partir de allí, la tesis de la infinitud actual de partes en el continuo. En lo siguiente mostraremos que, en la primera mitad de la década de 1670, Leibniz se ha representado una infinitud actual de partes en el continuo según el modelo de las series infinitas. Aunque quizás sea en última instancia irrelevante qué tipo de series haya tenido en mente al considerar la infinitud de partes del continuo, Leibniz inicialmente ha tenido en cuenta el primer modelo de infinito, esto es, aquel presente en las series que decrecen según una progresión geométrica. Aunque será examinado detalladamente en el siguiente apartado, es significativo en relación con el problema del continuo el hecho de que la suma de muchas de las series, sea del primer o del segundo tipo, dan por resultado cantidades finitas. Es significativo, decíamos, porque esto le ha permitido a Leibniz justificar sin absurdos que de un número actualmente infinito de partes no resulta una cantidad infinita en magnitud. De esta manera, por ejemplo, la suma de la serie que decrece geométricamente en mitades es $1 / 2+1 / 4+1 / 8$ etc. $=1 / 1$; similarmente, la suma de la serie de los recíprocos de los números triangulares es $1 / 1+1 / 3+1 / 6+1 / 10+1 / 15+1 / 21+1 / 28+$ etc. $=2$ (A II 1, 344). Así, aunque sean series de distinto tipo, lo importante es que para que una suma infinita dé como resultado un todo finito, las fracciones componentes deben responder a un orden de sucesión dado.

Veamos a continuación de qué manera los desarrollos de Leibniz de 1672 sobre series infinitas nos permiten representarnos la manera como habría entendido la infinitud actual de partes del continuo en la TMA. Anteriormente hemos señalado a la pasada que, para explicar las series en las cuales los términos son proporcionales, Leibniz se vale del recurso de exponerlas en líneas. Ahora bien, en los escritos en los que examina la tesis de la infinitud actual de partes en el continuo, sigue el mismo procedimiento, esto es, expone mediante una línea geométrica el continuo que está examinando. Así, por ejemplo, en el cuarto fundamento predemostrable de la TMA que citamos anteriormente puede verse que, en el intento de justificar que se dan indivisibles en el continuo, Leibniz lleva a cabo una división de una magnitud, representada en la línea $a b$, en partes proporcionales: «sea aquello cuyo inicio se busca expuesto en la línea $a b$,

Thémata. Revista de Filosofía №53 (2016) pp.: 289-310. 
cuyo punto medio es $c$, y sea $d$ el medio entre $a$ y $c$ y $e$ entre $a$ y $d$, y así sucesivamente» (A VI 2, 264). Mediante su exposición en una línea, Leibniz divide al continuo en partes que son cada una de ellas la mitad de la anterior, esto es, según la serie conocida como 'serie de Zenón' ${ }^{30}$ De esta manera, procede dividiendo primero la línea $a b$ en su mitad $a c$, para luego dividir esta sección nuevamente al medio, resultando de esta división $a d$, y así sucesivamente. De esta manera, $a b / a c=a c / a d=a d / a e=$ etc. , esto es, se respeta aquí la propiedad de las series mencionada anteriormente según la cual entre una subsección y una sección se mantiene la misma razón que entre dicha sección y el todo. Como en este caso cada subsección representa la mitad de la sección en la cual se incluye, $a c$ es $1 / 2 a b$, así como $a d$ es $1 / 2 a c$ y por eso mismo $1 / 4 a b$, del mismo modo que ae es $1 / 2 a d$ y $1 / 8 a b$, y así sucesivamente.

De esta manera, el hecho de exponer en líneas es una suerte de nexo epistemológico entre la aritmética y la física mediante la geometría, pues Leibniz expone en líneas (que son entidades geométricas) tanto series infinitas de números (entidades aritméticas) como un espacio, un cuerpo, un movimiento o un tiempo (entidades físicas). Dicho de otro modo, la manera de entender la división actual infinita del continuo fue para Leibniz idéntica a la partición de una línea geométrica que representa una serie infinita. Así, aquí se encontraría el 'núcleo matemático' del tratamiento leibniziano del continuo. Veamos el siguiente pasaje de un escrito de 1672 en el que también se considera la relación proporcional que se da entre el espacio, el tiempo y el movimiento:

Sea el espacio $a b$. Entiéndase que en él marcha el cuerpo $C$, con un movimiento uniforme, y atraviesa, en el espacio de una hora, de $a$ hasta $b$. Es necesario que, en el espacio de media hora, atraviese hasta $d$, y en el espacio de un cuarto [de hora] hasta $e$, y en un octavo [de hora] hasta f, y así dividiendo continuamente el espacio y el tiempo en la misma razón. (A VI 3, 81. La traducción es nuestra)

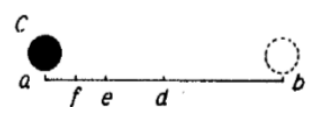

De acuerdo con Leibniz, dado un movimiento uniforme (es decir, uno que se mantenga constante, sin aumentar ni disminuir su velocidad a lo largo del trayecto recorrido), el espacio y el tiempo se dividirían en la misma razón. Por eso, la porción de espacio recorrido sería proporcional a la de tiempo transcurrido. En este sentido, hay una isomorfía entre el espacio y el tiempo que tiene como una de sus consecuencias que la geome-

[30] Arthur, R., "Leibniz's Inversion of Zeno", op. cit., y Arthur, Richard T. W., Leibniz, Cambridge: Polity Press, Classic Thinkers, 2014, pp. 79 y ss.

Thémata. Revista de Filosofía No53 (2016) pp.: 289-310. 
trización del espacio llevada a cabo por Leibniz acarrea necesariamente la del tiempo y, en fin, de los restantes continuos. Como se observa, la manera con la que Leibniz se representa dicha proporcionalidad no es otra que dividiendo el espacio y el tiempo en partes que decrecen al infinito con una progresión geométrica.

\section{III}

Esta manera de entender cómo Leibniz se habría representado una infinitud actual de partes del continuo en la $T M A$ tiene una importante consecuencia. Como se ha dicho anteriormente, en la Accessio Leibniz ha presentado una regla universal para la suma de series infinitas de términos. Leibniz muestra que las sumas de las series que decrecen al infinito según una progresión tanto geométrica como aritmética dan por resultado cantidades finitas (excepto la suma de la serie de las unidades y de los recíprocos de los números naturales que para Leibniz dan cantidades infinitas y que considera como imposibles o iguales a cero). Esto significa que, aunque no pueda hallarse un último término asignable de una serie, esto es, aunque ella sea infinita, de su agregación no resulta una magnitud infinita sino finita. Estos desarrollos de Leibniz fueron decisivos para el problema de la composición del continuo. Tomemos, como caso, la tesis leibniziana según la cual en cualquier cuerpo dado hay infinitas partes actuales (A VI $2,280)$. Es inevitable preguntarse cómo es que de una infinitud actual de partes no deviene un cuerpo infinito en extensión. ${ }^{31}$ Dado el «núcleo matemático» de los desarrollos leibnizianos relativos al problema del continuo, veremos que Leibniz respondería a este inconveniente diciendo que de la infinita agregación de partes no deviene un cuerpo infinito si las partes son interpretadas según alguno de los dos modelos que han sido expuestos previamente.

Consideremos esta cuestión, en primer lugar, en relación con la suma de las series que decrecen al infinito según una progresión geométrica. Leibniz extrae una consecuencia de la propiedad de estas series men-

[31] Este fue uno de los argumentos que Gregorio de Rimini (ca. 1300-1358) planteó frente a una composición atomista y que fue retomado por Froidmont, L., Labyrinthus sive de compositione continui, op. cit., 98. Véase nuestra ponencia «G. W. Leibniz y L. Froidmont: coincidencias en sus abordajes del problema del continuo» presentada en el II Congreso Internacional de la Sociedad Filosófica del Uruguay, cuyas actas pueden hallarse aquí: http:// sfu.org.uy/sfu_blog/?p=564 [última consulta: 22/04/2015]

Thémata. Revista de Filosofía $\mathrm{N}^{\circ} 53$ (2016) pp.: 289-310. 
cionada anteriormente, que entre una sección y el todo se mantiene la misma ratio que entre una subsección y una sección, a saber:

Supuestas las Fracciones continuamente decrecientes cuyo numerador sea la unidad, pero cuyos Denominadores sean los términos de alguna progresión geométrica, la suma de todas las Fracciones de una progresión dada será la primera Fracción de la progresión Geométrica precedente». (A II 1, 344)

Esta regla expresa, ante todo, que para sumar una serie de este tipo cualquiera se requiere del conocimiento de otras series decrecientes geométricamente. De esta manera, para la suma de estas series debe establecerse, en primer lugar, el orden las series que decrecen de este modo. Dicho orden se determina de acuerdo con la ratio con la que se generan cada una de las series. En este sentido, basta con la consideración de la primera de las fracciones para reconocer el orden. Así, por ejemplo, la serie que se genera de acuerdo con la razón de $1 / 3$ se encuentra, en el orden de las series, luego de la que se genera de acuerdo con la razón de $1 / 2$ y antes de la que sigue la razón de $1 / 4$. De esta manera:

Primera serie: $1 / 2,1 / 4,1 / 8,1 / 16$, etc.

Segunda serie: $1 / 3,1 / 9,1 / 27,1 / 81$, etc.

Tercera serie: $1 / 4,1 / 16,1 / 64,1 / 256$, etc.

$\mathrm{Y}$ así sucesivamente.

Establecido el orden de las series, a modo de ejemplo, intentemos sumar a continuación la tercera serie. De acuerdo con la regla antes mencionada, la suma de esta serie da por resultado la fracción que es el primero de los términos de la serie precedente, es decir que 1/4, 1/16, 1/64, 1/256, etc. $=1 / 3$. Por la misma razón, $1 / 3,1 / 9,1 / 27,1 / 81$, etc. $=1 / 2$ y 1/2, 1/4, 1/8, 1/16, etc. $=1 / 1$ (donde $1 / 1$ sería, de acuerdo con el procedimiento señalado, el primer término de la serie precedente) (A II 1, 344). De esta manera, puede observarse que las sumas de las series que decrecen al infinito según una progresión geométrica dan por resultado las magnitudes finitas expresadas en las respectivas fracciones.

Para la suma de las series que decrecen al infinito según una progresión aritmética, Leibniz presenta una regla que sigue un procedimiento relativamente análogo al de la anterior. No obstante, de acuerdo con el filósofo de Leipzig él es el primero que ha ofrecido una regla para sumar estas series (A II 1,344). Por eso, en gran medida toda la Accessio está orientada a la presentación de esta regla. Dicha regla de Leibniz para la suma de series que decrecen según una progresión aritmética al infinito es:

(...) la suma de las razones en las que el antecedente es la Unidad y el consecuente es un término de una progresión Aritmética Replicada (...); esta suma, digo, es una fracción o razón cuyo numerador o antecedente es el exponente de la serie precedente 
inmediata, es decir, de la penúltima (supuesta, a saber, una última dada), pero el denominador o consecuente es el exponente de la serie precedente inmediata a la precedente, esto es, de la antepenúltima». (A II 1, 346)

Como esta regla remite a «la serie precedente inmediata» y a «la serie precedente inmediata a la precedente», es claro que Leibniz también piensa en este caso un orden de las series que decrecen según una progresión aritmética. Dicho orden no es otro que el expuesto en el triángulo de Pascal. El mismo orden se conserva cuando las series consideradas son las de los respectivos recíprocos, esto es:

Recíprocos de los números naturales: $1 / 1,1 / 2,1 / 3,1 / 4$, etc.

Recíprocos de los números triangulares: $1 / 1,1 / 3,1 / 16,1 / 10,1 / 15$, etc.

Recíprocos de los números piramidales: $1 / 1,1 / 4,1 / 10,1 / 20$, etc.

Recíprocos de los números triángulo-triangulares: 1/1,1/5, 1/15, 1/35, etc.

Recíprocos de los números triángulo-piramidales: 1/11,1/1/1/21, 1/56, etc.

Y así sucesivamente.

Para la comprensión de la regla dada por Leibniz para la suma de estas series, es necesario explicar qué entiende por el «exponente». Sintéticamente, el exponente de una serie se expresa en el denominador del segundo término de cada una de las series, esto es, de la fracción que sigue a la de la unidad. De esta manera, el exponente de la serie de los números naturales es 2 , del mismo modo que el de los triangulares es 3 , y así sucesivamente (A II 1, 346). De acuerdo con la regla de Leibniz, entonces, la suma de cualquiera de estas series da por resultado una fracción en la que el nominador es el exponente de la serie anterior y el denominador el exponente de la serie que antecede a la anterior. Por lo tanto:

Suma de los recíprocos de los triangulo-piramidales: 5/4

Suma de los recíprocos de los triangulo-triangulares: $4 / 3$

Suma de los recíprocos de los piramidales: $3 / 2$

Suma de los recíprocos de los triangulares: $2 / 1$

De esta manera, Leibniz demuestra que la suma de los infinitos términos que componen la serie de los recíprocos de los números triangulares (como un caso de serie que decrece al infinito según una progresión aritmética) es 2 , es decir, una magnitud finita. De este modo, siguiendo este modelo de comprensión del infinito actual, lo mismo que en relación con el modelo analizado anteriormente, puede pensarse una infinitud actual de partes 'dentro de' una magnitud de hecho finita.

Como hemos visto, en la TMA Leibniz expresa las magnitudes continuas, como el espacio, el tiempo, el cuerpo y el movimiento, en líneas que se dividen de acuerdo con una progresión geométricamente decreciente al infinito. Precisamente por el hecho de que Leibniz se representa los continuos de esta manera, las propiedades o características que hemos 
mencionado de las series que decrecen según los dos modelos explicados se aplicarían en la consideración de los continuos. Esto significa que, así como la suma de una serie que posee infinitos términos es una cantidad finita, así también las infinitas partes de, por ejemplo, un cuerpo cualquiera (y lo mismo vale para un espacio, un tiempo o un movimiento) componen un cuerpo no infinito sino finito en extensión. De esta manera, Leibniz habría encontrado en las series unos modelos matemáticos con los cuales explicar la infinitud actual de partes del continuo.

\section{Conclusiones}

En este trabajo nos propusimos mostrar que los exámenes sobre series infinitas que Leibniz ha llevado a cabo en el año 1672 pueden ayudarnos a esclarecer la manera como ha concebido la tesis de la infinitud actual de partes en el continuo afirmada en la TMA del año anterior. Como también hemos visto, esta interpretación se opone a la concepción según la cual en la TMA Leibniz habría entendido que el continuo se compone de indivisibles. Para finalizar quisiéramos mencionar dos cuestiones vinculadas con nuestra propuesta.

La primera cuestión, que nos permitirá ver la interpretación que proponemos en toda su dimensión, está relacionada con el alcance de la tesis leibniziana de la infinitud actual de partes en el continuo. En nuestro trabajo hemos visto que podría entenderse que en la TMA Leibniz habría comprendido que en el espacio, el tiempo, el cuerpo y el movimiento hay infinitas partes actuales entendidas como decrecientes según una progresión geométrica que, a su vez, poseen extremos indivisibles. Ahora bien, como todas las infinitas partes representadas en fracciones son por eso mismo asignables, ellas poseen, a su vez, una infinitud actual de partes. En este sentido, la consideración de la estructura interna de cada una de dichas partes debe replicar el mismo modelo de comprensión de un infinito actual en partes proporcionales antes mencionado. En consecuencia, en una parte de un espacio, un cuerpo, un tiempo o un movimiento cualquiera se da proporcionalmente la misma estructura que se halla en dicho espacio, cuerpo, tiempo o movimiento. De esta manera, cada una de las partes debe ser tomada como dividida según una misma razón. ${ }^{32}$ La manera como re-

[32] Como han señalado Beeley, Ph., Kontinuität und Mechanismus, op. cit., 197-198; Arthur, R., The Labyrinth of the Continuum, op. cit., xxxii, para Leibniz las observaciones microscópicas, especialmente de Hooke y Kircher, han significado un gran apoyo para la tesis

Thémata. Revista de Filosofía $\mathrm{N}^{\circ} 53$ (2016) pp.: 289-310. 
presentarse un infinito actual en el continuo se replica al infinito, esto es, en cada una de las infinitas partes. Dicho en términos de series infinitas, a modo de ejemplo, la primera de las fracciones de la serie de Zenón ( $1 / 2,1 / 4,1 / 8$, etc.) es asimismo la suma de una serie infinita de fracciones (esto es, de $1 / 3,1 / 9,1 / 27$, etc.). En este sentido, hay 'series dentro de series al infinito'. Podemos referirnos a esta comprensión del continuo utilizando una denominación que, aunque sea muy posterior a Leibniz, representa a la perfección la intención del autor: la estructura del continuo leibniziano de la TMA es fractal. Con esto nos referimos no a la teoría fractal sino específicamente al aspecto que adquieren las figuras fractales, esto es, a la estructura geométrica fractal en la que siempre se repite el mismo patrón. Esto significa que, de ser correcta nuestra interpretación, el espacio, el cuerpo, el movimiento y el tiempo poseen una configuración particionada según una progresión geométrica decreciente al infinito que se repite en diversas escalas de grandeza o pequeñez. Antes que nosotros, Samuel Levey ha reconocido que los exámenes de Leibniz, particularmente los relativos al movimiento, incluyen la comprensión de una estructura fractal. ${ }^{33}$ No obstante, sus análisis recaen sobre los desarrollo leibnizianos de 1676. Lo que nosotros proponemos, entonces, es que la comprensión fractal de la estructura del continuo leibniziano es anterior a los pensamientos de 1676, pudiéndose retrotraer hasta 1671.

La segunda cuestión que queremos abordar a modo de conclusión es que, si nuestra interpretación es correcta, Leibniz no habría sido el primero en sostenerla. Más aún, Libert Froidmont, un pensador históricamente anterior a Leibniz, habría entendido la composición del continuo de una manera muy similar -aunque no idéntica-en su obra Labyrinthus sive de compositione continui de 1631. Si bien excede por completo la intención de nuestro trabajo introducirnos en la obra de Froidmont, ${ }^{34}$ veamos,

del infinito actual (A VI 2, 241).

[33] Levey, Samuel, “The Interval of Motion in Leibniz's Pacidius Philalethi”, en NOÛS, 47, 3, 2003, pp. 371-416.

[34] Puede hallarse una exposición de la relación entre el pensamiento de Froidmont y el de Leibniz en lo que respecta al problema del continuo en Beeley, $\mathrm{Ph}$., Kontinuität und Mechanismus, op. cit., capítulo 12. Por nuestra parte, hemos presentado una breve aproximación a la similitud que hay entre la manera como ambos autores han entendido la composición del continuo en «Indivisibles y movimiento en el continuo leibniziano» que se publicará en el número 45 del año 2015 de la Revista Latinoamericana de Filosofía, así como también en nuestra ponencia «G. W. Leibniz y L. Froidmont: coincidencias en sus abordajes del problema del continuo» referida anteriormente.

Thémata. Revista de Filosofía $\mathrm{N}^{\circ} 53$ (2016) pp.: 289-310. 
a modo de ejemplo, un pasaje de su obra en el que puede observarse una proximidad en relación con el planteo de Leibniz:

Puesto que en cualquier parte del continuo existen otras infinitas partículas menores y que cada una comprende infinitas unidades de otras partes, etc., es evidente, por consiguiente, que en cualquier parte existen otras infinitas partículas infinitésimas [particulae aliae infinities infinitae]. Todas estas cosas se siguen muy cierta y evidentemente supuesta aquella admirable infinitud de partes. ${ }^{35}$

Incluso, hay una razón de la proximidad entre los planteos de Leibniz y Froidmont. En efecto, Leibniz conocía la obra de Froidmont al menos desde el mismo año en que redactó la TMA (A II 1, 111). ${ }^{36}$ No obstante, una de las diferencias teóricas entre Leibniz y Froidmont es el hecho de que este último no reconoce extremos indivisibles en el continuo, ${ }^{37}$ cosa que es esencial en el planteo de Leibniz para explicar la unidad o cohesión de los cuerpos (A VI 2, 266). Froidmont podría estar oponiéndose, presumiblemente, a la interpretación de aquellos como Francisco Suárez para quienes han de admitirse puntos que justifiquen la continuidad de las partes (puncta continuantia). ${ }^{38}$ Sea como fuere, es importante que, en el mismo trasfondo histórico de Leibniz donde se encuentran interpretaciones como la de Galileo, para quien el continuo se compone de indivisibles, se hallen también otras interpretaciones como la de Froidmont que reconoce infinitas partes que no son indivisibles sino proporcionales.

\section{Bibliografía}

Andersen, Kirsti, “Cavalieri's Method of Indivisibles”, en Archive for History of Exact Sciences, 31, 1985, pp. 291-367.

Andersen, Kirsti, "The Method of the Indivisibles: Changing Understandings", en Studia Leibnitiana, Sonderheft 14, 1986, pp. 14-25.

[35] Froidmont, L., Labyrinthus sive de compositione continui, op. cit., 171. Traducción nuestra. [36] En la reseña de la obra de Beeley Kontinuität und Mechanismus que ha hecho R. Arthur (Arthur, R., Mercer, C., Smith, J. y Wilson, C., "Symposium on Philip Beeley's Kontinuität und Mechanismus" (reseñas de la obra por dichos autores), Leibniz Society Review, Vol. 7, 1997, pp. 27-28), el autor señala que lo que movilizó a Beeley a dedicar buena parte de su libro a la obra de Froidmont fue una breve indicación que Leibniz realizó en el prefacio de la Theoria motus abstracti de 1671 al «laberinto del continuo y el movimiento», aunque no obstante también indica que la primera vez que Leibniz explícitamente se refirió al libro de Froidmont tuvo lugar en el año 1676. El hecho de que Leibniz se haya referido explícitamente a la obra de Froidmont en el período en que redactó la TMA es, en consecuencia, muy relevante, pues deja abierta la posibilidad de que la influencia del teólogo lovaniense en Leibniz haya sido mucho mayor que la que hasta aquí se ha considerado.

[37] Froidmont, L., Labyrinthus sive de compositione continui, op. cit., 104.

[38] Suárez, F., Opera omnia, op. cit. 559.

Thémata. Revista de Filosofía N53 (2016) pp.: 289-310. 
Arthur, Richard T. W., "Leibniz on Continuity", en PSA:Proceedings of the Biennial Meeting of the Philosophy of Science Association, 1, 1986, pp. 107-115.

Arthur, R., Mercer, C., Smith, J. y Wilson, C., "Symposium on Philip Beeley's Kontinuität und Mechanismus" (reseñas de la obra por dichos autores), Leibniz Society Review, Vol. 7, 1997, pp. 25-64.

Arthur, Richard T. W., "Cohesion, Division and Harmony: Physical Aspects of Leibniz's Continuum Problem (1671-1686)", en Perspectives of Science, 6 nos. 1 \& 2, 1998, pp. 110-135.

Arthur, Richard T. W., "Leibniz's Inversion of Zeno: Continuity of Motion, Substantial Action and Plurality", en Workshop 'Corporeal Substances and the Labyrinth of the Continuum' (2000), http://www. humanities.mcmaster.ca/ rarthur/papers/LIZ.pdf Consultado: 10/02/2016] Arthur, Richard T. W., The Labyrinth of the Continuum. Writings on the Continuum Problem, 1672-1686, textos seleccionados, traducidos y editados e introducción elaborada por Richard T. W. Arthur, New Haven y Londres: Yale University Press, 2001.

Arthur, Richard T. W., "Actual Infinitesimals in Leibniz's Early Thought", en The Philosophy of the Young Leibniz, Studia Leibnitiana, Sonderheft 35 2009, pp. 1-26.

Arthur, Richard T. W., Leibniz, Cambridge: Polity Press, Classic Thinkers, 2014.

Bassler, Otto Bradley, "The Leibnizian Continuum in 1671", en Studia Leibnitiana, 30, 1, 1998a, pp. 1-23.

Bassler, Otto Bradley, "Leibniz on the Indefinite as Infinite", en The Review of Metaphysics, 51, 4, 1998b, pp. 849-874.

Beeley, Philip, Kontinuität und Mechanismus. Zur Philosphie des jungen Leibniz in ihrem Ideengeschichtlichen Kontext, Studia Leibnitiana Supplementa, vol. 30, Stuttgart: Franz Steiner Verlag, 1996.

Beeley, Philip, "Mathematics and nature in Leibniz's early philosophy", en Brown, Stuart ed., The young Leibniz and his philosophy (1646-76), Dordrech/ Boston/London : Kluwer Academic Publishers, 1999, pp. 123-145.

Beeley, Philip, "Infinity, Infinitesimals and the Form of Cavalieri: John Wallis and his Critics", en Ursula Goldenbaum y Douglas Jesseph eds., Infinitesimal Differences: Controversies between Leibniz and his Contemporaries, Berlin y New York: Walter de Gruyter, 2008, pp. 31-52.

Bernstein, Howard, "Conatus, Hobbes, and the young Leibniz", en Studies in History and Philosophy of Science, 11, 1, 1980, pp. 25-37. Boyer, Carl B., The history of the calculus and its conceptual developement, New York: Dover Publications, Inc., 1949.

Cavalieri, Bonaventura, Geometria indivisibilibus continuorum quadam nova ratione promota, Bononiae, 1653 [1635]. 
Descartes, René, Oeuvres de Descartes, publicadas por Charles Adam y Paul Tannery, París: Vrin, 1964-1974 (citado como AT).

Euclides, Elementos. Libros I-IV (introducción de Luis Vega, traducción y notas de María Luisa Puertas Castaños), Madrid: Gredos, 1991.

Froidmont, Libert, Labyrinthus sive de compositione continui, Anvers, 1631.

Galilei, Galileo, Le opere di Galileo Galilei, Florencia, Edizione Nazionale, Vol. VIII, 1898 (Citado como EN).

Gassendi, Pierre, Opera omnia in sex tomos divisa, Lvgdvni: Sumptibus Lavrentii Anisson \& Ioan. Bapt., Devenet, 1658, tomo primero.

Hofmann, Joseph E., Leibniz in Paris, 1672-1676. His growth to mathematical maturity, Cambridge y New York: Cambridge University Press, 1974.

Hobbes, Thomas, Opera Philosophica quae latina scripsit omnia, Londres: Bohn, 1839-1845.

Lawrenz, Jürgen, The Nature of Reality and the Reality of Nature. A Study of Leibniz's Double-Aspect Ontology and the Labyrinth of the Continuum, Cambridge : Cambridge Scholars Publishing, 2010.

Leibniz, G. W., Sämtliche Schriften und Briefe, Darmstadt; Leipzig; Berlin: Akademie-Verlag, 1923 y ss. (Citado como A, seguido de la serie en números romanos, el tomo en números arábigos y finalmente el número de página)

Leibniz, G. W., Quadrature arithmétique du cercle, de l'ellipse et de l'hyperbole et la trigonométrie sans tables trigonométriques qui en est le corollaire (introducción, traducción y notas de Marc Parmentier, texto latino editado por Ebenhard Knobloch), París: Vrin, 2004.

Leibniz, G. W., Obras filosóficas y científicas, Granada: Editorial Comares, 2007 y ss. (Citado como OFC, seguido del volumen y número de página).

Levey, Samuel, "The Interval of Motion in Leibniz's Pacidius Philalethi", en NOÛS, 47, 3, 2003, pp. 371-416.

Lison, Elad, "The Philosophical Assumptions Underlying Leibniz's Use of the Diagonal Paradox in 1672", en Studia Leibnitiana, 38, 2, 2006, pp. 197-208.

López, E. y Graña, M. eds, Sobre los principios de la filosofía (traducción y notas), Madrid: Gredos, 1989.

Pascal, Blaise, Traité du triangle arithmétique, avec quelques autres petits traitez sur la mesme matière, París: G. Desprez, 1665.

Suárez, Francisco, Opera omnia, vol. XXVI, Apud Ludovicum Vives, Bibliopolam Editores, París. 1861. 